\title{
Einstein's Theory of Relativity in the Scenario of the Higgs Quantum Space Dynamics
}

\author{
Jacob Schaf \\ Universidade Federal do Rio Grande do Sul (UFRGS), Instituto de Fsica, Porto Alegre, Brazil \\ Email: schaf@if.ufrgs.br
}

How to cite this paper: Schaf, J. (2018) Einstein's Theory of Relativity in the Scenario of the Higgs Quantum Space Dynamics. Journal of Modern Physics, 9, 1111-1143.

https://doi.org/10.4236/jmp.2018.95068

Received: March 24, 2018

Accepted: April 27, 2018

Published: April 30, 2018

Copyright $\odot 2018$ by author and Scientific Research Publishing Inc. This work is licensed under the Creative Commons Attribution International License (CC BY 4.0).

http://creativecommons.org/licenses/by/4.0/

Open Access

\begin{abstract}
This work is concerned about the true physical mechanism of gravity. The Higgs theory introduces important changes in Einstein's view about the nature of empty space, about the meaning of motions and about the nature of the gravitational physics. The Higgs Quantum Space (HQS) is a real quantum fluid spatial medium, giving inertial mass to the elementary particles by the Higgs mechanism. Therefore, the HQS rules the inertial motion of matter-energy and is the local ultimate reference for rest and for motions. In this new scenario, velocity with respect to the local HQS and not relative velocity is the origin of all the effects of motion. The HQS also necessarily is responsible for the gravitational fields because it is mass that creates the gravitational fields. The observed absence of the gravitational slowing of the GPS clocks by the solar field and the absence of light anisotropy with respect to earth are both the obvious signature of the true physical mechanism of gravity in action. These observations show that the HQS is moving itself round the sun according to a Keplerian velocity field, consistent with the planetary motions, which will be shown to accurately create, besides the observed gravitational dynamics, all the observed effects of the gravitational fields on light and on clocks. Current theories explain the absence of the solar gravitational slowing of the GPS clocks with base in the principle of equivalence. In the language of the present work, the HQS materializes the local Lorentz frames (LFs), turning them into local proper LFs, intrinsically stationary with respect to the local moving HQS. In its motions, the HQS carries these local proper LFs with it round the sun, so that the planets of the solar system are stationary with respect to them, which directly predicts the absence of the gravitational slowing of the GPS clocks by the solar field, the absence of light anisotropy with respect to earth and all the other effects of the gravitational fields observed on earth.
\end{abstract}




\section{Keywords}

Fundamental Physics, Theory of Relativity, Relativistic Effects, Time Dilation, Gravitational Time Dilation, Higgs Theory, Relativistic Experiments

\section{Introduction}

\subsection{Briefly the Foundations of Special Relativity}

Since Galileo, it was well-known that local mechanical experiments are unable to detect the velocity of a laboratory in uniform motion along a straight line. This observational fact has the consequence that the laws of mechanics are invariant with changes of the inertial reference, which is known as Galilean invariance. In Einstein's view the fact that the Michelson light anisotropy experiments gave null results, in spite of the visible motion of earth, demonstrates that local electromagnetic experiments too cannot reveal the state of motion of the earth-based laboratories. In order to put this idea in practice, he assumed that, in the view of a hypothetical stationary observer, the rate of clocks and the lengths of the meter sticks change with relative velocity in such a way that they let invariant the laws electrodynamics. This has enforced the conclusion that measurements of the velocity of light, by the method of light go-return round-trips in any direction, give the same result. He concluded that the velocity of light is intrinsically constant and isotropic with respect to every possible inertial reference. He also has extended the invariance with changes of the inertial reference to all the laws of physics, which he named Principle of Relativity [1] [2].

By using clever thought experiments, he obtained the general laws of transformation for space and time coordinates that accomplish his postulates. They are the well-known Lorentz transformations:

$$
\begin{gathered}
x^{\prime}=\frac{x-v_{r} t}{\sqrt{1-v_{r}^{2} / c^{2}}} \\
y^{\prime}=y \\
z^{\prime}=z \\
t^{\prime}=\frac{t-v_{r} x / c^{2}}{\sqrt{1-v_{r}^{2} / c^{2}}}
\end{gathered}
$$

where $1 /\left(1-v^{2} / c^{2}\right)^{1 / 2}=\gamma$. These transformations let the form of the laws of physics invariant with changes of the inertial reference, which is known as Lorentz invariance of the laws of physics.

Many well-known experiments, testing the predictions of theory of relativity (TR) on earth along the whole past century, systematically have confirmed again and again the predictions of the TR. However, in the present work, it will become clear that earth is a very specific preferred reference, closely stationary 
with respect to the local Higgs Quantum Space (HQS), giving inertial mass to the elementary particles and hence being the ultimate reference for rest and for motion of matter-energy. Therefore, the earth-based references, for all practical instances, are not hypothetically however truly stationary preferential references. Besides this, it was soon discovered that the reciprocal symmetry between the observers, which is an intrinsic characteristic of the TR, leads to many unsolvable paradoxes. Also Einstein tried to explain the origin of inertial mass of matter bodies in terms of Mach's ideas. This explanation however opened more problems than it solved. A scientifically sound explanation for the origin of the inertial mass was found only fifty years later by the Higgs theory.

\subsection{The Central Ideas of General Relativity}

In the General Theory of Relativity (GR) [1] [2], Einstein has declined Newton's theory of gravitation, because it explains gravity in terms of gravitational forces that cannot be real. In his GR he provides a completely new theory of gravitation, in which the gravitational dynamics is the result of generalized inertial motions (geodesic motion) of the matter bodies in the curved four-dimensional spacetime. According to the principle of equivalence, these geodesic motions cancel the effects of the gravitational field in these local references. In Einstein's view, a free falling elevator is an inertial reference (IR). He assumed that in the gravitational fields the local references, free-falling from the infinite at the local escape velocity, are local proper references. It however is well-known that, in a gravitational field, each local IR is valid at only one point of space and that any two IRs, not located at exactly the same position, are mutually non-inertial. Hence, Einstein's free-falling elevator is a different IR at each point of space along its path. It scans through an infinite sequence of local IRs. The fact that, in the view of an observer in the free-falling elevator, everything apparentlybehaves as in a true IR is simulated by the fact that the elevator and the observer in it are under identical kinematical circumstances and his instruments are not sensitive enough to detect the small discrepancies between the different points in the elevator.

In order to explain the orbital motion of the planets in the absence of Newtonian gravitational forces, Einstein assumed that the astronomical bodies curve the geometry of the four-dimensional spacetime in their neighborhood and that, in this curved spacetime, the orbits of matter bodies follow geodesic paths, governed by a generalized principle of inertia. He described this spacetime curvature by his famous field equations:

$$
G_{\mu \nu} \equiv R_{\mu \nu}-\frac{1}{2} g_{\mu \nu} R=8 \pi G T_{\mu \nu}
$$

Equation (2) is an equation for the metric tensor $g_{\mu v}$ of the curved spacetime, $R_{\mu v}$ is the Ricci curvature tensor, $R$ is the scalar curvature and $G$ is the gravitational constant. 
In the neighborhood of the gravitational sources, this curved spacetime is characterized by the invariant length of the four-dimensional line element. For sufficiently weak gravitational fields, its form is:

$$
\mathrm{ds} s^{2} \approx\left[1-\frac{2 U}{c^{2}}\right]^{-1} \mathrm{~d} r_{0}^{2}+r^{2} \mathrm{~d} w^{2}-c^{2}\left[1-\frac{2 U}{c^{2}}\right] \mathrm{d} t_{0}^{2}
$$

The coefficients $\left(1-\frac{2 U}{c^{2}}\right)^{-1}$ and $-c^{2}\left(1-2 U / c^{2}\right)$ are respectively the diagonal $g_{11}$ and $g_{44}$ components of the Schwarzschild metric tensor. The last term of this equation expresses the gravitational time dilation as viewed by an external observer, where $\mathrm{d} t_{0}$ is an infinitesimal time interval in the absence of a gravitational field, $\mathrm{d} r_{0}$ is an infinitesimal interval of radial distance in the absence of a gravitational field and $2 U=2 G M / r$ is the square of the local escape velocity from the gravitational field. According to the last term, the rate of clocks, stationary within the gravitational field, run slow because the period of the time standard $T$, by which the clock counts time, is longer by a factor $\left(1-2 U / c^{2}\right)^{-1 / 2}$, given by:

$$
T(r)=T_{0}\left(1-2 U / c^{2}\right)^{-1 / 2}
$$

where $T_{0}$ is the time period for $(U=0)$. Also, the velocity of light directly toward the gravitational center, from the viewpoint of an external observer, will seem to be gradually reduced according to $c^{\prime}(r)=\left(c^{2}-2 G M / r\right)^{1 / 2}$ and the rate of clocks will seem to be gradually slowed according to Equation (4). Near to the event horizon of a black hole they will both seem to stop completely.

In Equation (3), the last term is the square of the effective time coordinate that can be written as $\left(c^{2}-2 U\right) \mathrm{d} t_{0}^{2}$, where $t_{0}$ is the time rate for $U=0, c^{2}-2 U$ is the square of the local effective velocity along the time axis in a gravitational field. As the velocity of light $c$, in Einstein's spacetime, is intrinsically orthogonal to the local escape velocity $(2 U)^{1 / 2}$, the effective velocity can be obtained by the formula of Pythagoras $c^{\prime}=\left(c^{2}-2 U\right)^{1 / 2}$. This term is usually interpreted as a gravitational slowing of the clock rate. In the first term of Equation (3), the time $\mathrm{d} t$ for a light pulse to travel a distance $\mathrm{d} r$ along the radial coordinate can be written as $\mathrm{d} t=\mathrm{d} r / c=\mathrm{d} r_{0} /\left(c^{2}-2 U\right)^{1 / 2}$, where $c^{\prime}=\left(c^{2}-2 U\right)^{1 / 2}$ again is the effective velocity of light, however along the radial coordinate. As, from the viewpoint of the theory of relativity, the velocity of light is intrinsically constant $\left(c^{\prime} \equiv c\right)$, this gradual reduction of the velocity of light, is imputed to stretching of the radial distances and not to reduction of the effective radial velocity component of light. From the present viewpoint, GR expresses the radial distances $\mathrm{d} r$ in units of the local effective velocity of light.

\subsection{Discussion}

Experimental observations have shown that atomic clocks, stationary within a gravitational field, corroborate, to first order of approximation, exactly the gravitational slowing predicted by GR. However, recent experimental observations 
have revealed that the GPS clocks, moving with earth round the sun, do not show the gravitational slowing by the solar field. As the local escape velocity is an intrinsic characteristic of the gravitational field that has a well-defined value at each point and cannot depend on the motion of the clock, this observation runs into conflict with Equation (4). It demonstrates that the orbital velocity of earth cancels locally the effects of the solar gravitational field. The earth's orbital motion restores the velocity of light to its value in free space (vacuum). This cannot be explained within the original scenario of GR.

Current theories explain the absence of the solar gravitational slowing of the GPS clocks in terms of the principle of equivalence [3]. Accordingly, the local Lorentz frame (LF) of earth is a local proper LF in which the orbiting earth as well as the GPS clocks, moving with it, are stationary. It is assumed that the gravitational slowing is absent, because in the local reference of earth, the GPS clocks are free-falling with earth in the solar field, this way canceling the effects of the solar field. This will say that, in the local reference of earth, spacetime is flat, the solar gravitational pull is zero and all the effects of the solar gravitational field on light and on clocks are canceled. However, these same GPS clocks too are moving along circular orbits round earth that too is closely spherically symmetric and hence, from the above viewpoint, they too are free-falling with the GPS satellites in the earth's field, which too should cancel the gravitational slowing by the earth's field. However, notwithstanding these same theories include, for the time rate of the GPS clocks, the gravitational slowing by the earth's field of $1.67 \times 10^{-10} \mathrm{sec} / \mathrm{sec}$, which clearly runs into conflict with the previous conclusions, with base in the principle of equivalence in the case of the solar field.

The point here is not putting in doubt the correctness of the calculated slowing of the GPS clocks by the earth's field, however to demonstrate that its explanation, simply in terms of the principle of equivalence, is not consistent. In reality, the gravitational slowing of the GPS clocks, in orbit round earth, was calculated, simply by taking into account the altitude and some presumed special relativistic and Doppler effects [3]. The fallacy in explaining the absence of the gravitational slowing of the GPS clocks by the solar field in terms of the principle of equivalence lies exactly in the assumption that any locally non-accelerated reference can be taken as a proper reference. This assumption is a landmark in the foundations of Einstein's theory of relativity that is propped up by the idea that empty space contains nothing that can be a reference for rest and for motions or to be a medium for the propagation of light. This assumption however was made without knowing the true nature of the empty space and the origin of the inertial mass of the elementary particles. The gravitational slowing will be shown in subsequent Sections to be due to a velocity, however, not relative velocity. It is due to a velocity of a more fundamental nature, which, in the solar system, is canceled by the orbital motion of earth along a direct circular equatorial orbit round the sun and which however is not canceled by the motion of 
the GPS clocks round earth in orbits inclined 55 degrees to the earth's equator.

The next Section 2 implements Einstein's original equivalence of gravitational and inertial effects in the gravitational fields and obtains natural and consistent explanations for all these observations, within the scenario of the Higgs Quantum Space (HQS) dynamics. This HQS, besides providing the scientifically sane origin of the inertial mass, its motion according to a certain velocity field, also creates the physical origin of the gravitational dynamics. This HQS dynamics will be seen to straightforwardly predict the absence of the gravitational slowing of the GPS clocks by the solar field, the absence of light anisotropy with respect to earth and all the other observed effects of the gravitational fields on light and on clocks. Importantly, it also straightforwardly predicts the observed non-cancelation of the gravitational slowing of the GPS clocks, moving round earth along non-equatorial orbits. These observations altogether will be seen to be the authentic signature of the true physical mechanism of gravity in action that Einstein has missed.

Current theories also affirm, with base in the principle of equivalence that a vertically free-falling clock does not run slow, because the effects of the gravitational field are locally canceled. By the same fallacious assumption as before, a free-falling reference is then seen as a proper Lorentz frame. Unfortunately, to now no experimental observation has tested the veracity of this assumption and hence it is an open question. According to these theories, when the free-falling clock is stopped, it will begin to run slow, according to Equation (4). The reason appointed is the fact that it acquires an implicit upward velocity (implicit because it is not with respect to the ordinary space) equal to the local escape velocity $(2 G M / r)^{1 / 2}$ with respect to the local free-falling inertial references, supposedly proper Lorentz frames. It is important to note here that accelerations do not cause any time dilation. This has been tested by Muon decay in cyclotrons for accelerations up to $10^{19} \mathrm{~m} / \mathrm{sec}^{2}$ [4]. Only their ordinary velocity causes time dilation.

A clock, moving in a circular orbit in the spherically symmetric gravitational field of the sun, obviously is not free-falling $(\mathrm{dr} / \mathrm{d} t=0)$ and, within the model of the free-falling inertial references, it in fact is implicitly moving upward at the local escape velocity $\left((2 G M / r)^{1 / 2}\right)$ with respect to each local inertial reference (falling from the infinite) along its path. Therefore, the GPS clocks, besides running slow, due to this implicit upward escape velocity, should in addition run slow, due to the orthogonal horizontal orbital velocity through the local free-falling inertial references. On the other hand, a clock, falling freely from a finite altitude, is not stationary with respect to Einstein's free-falling inertial references from the infinite. Therefore, clocks, falling freely from a finite altitude, must run slow (not showing proper time) in spite of being stationary within their own local free-falling reference (free-falling elevator), which, according to the principle of equivalence, too cancels locally the effects of the gravitational field.

The model of the free-falling inertial references has other grave troubles. It, 
for instance, cannot give rise to a gravitational pull. It cannot because the velocity of Einstein's vertically free-falling inertial references from the infinite, is constant at any fixed point $\left(r_{0}, \theta_{0}, \phi_{0}\right)$ so that, at each fixed point, the acceleration is zero $\left(\mathrm{d} v /\left.\mathrm{d} t\right|_{r_{0}}=0\right)$. A velocity, having a constant value with time and having a fixed direction, cannot give rise to an inertial pull. Therefore, a matter body, brought to rest at any fixed point $\left(r_{0}, \theta_{0}, \phi_{0}\right)$ and then released, should stay in equilibrium there, simply passing from one local free-falling inertial reference to the other identical one, which are all perfectly equivalent, without changing its velocity. Any perturbation however will initiate a runaway departure, upward or downward. In order to enable Einstein's model of the free-falling inertial references to produce a gravitational pull and initiate a free-fall, it would be necessary that the acceleration of the free-fall velocity at any fixed position $r_{0}$ be given by $\mathrm{d} v /\left.\mathrm{d} t\right|_{r_{0}}=g\left(r_{0}\right)$. This however would rapidly grow up the local free-fall velocity beyond the velocity of light, which clearly cannot be reasonable.

In a real gravitational field, in which the gravitational dynamics is the result of purely inertial motions (as required by GR), all matter bodies, not impeded to follow strictly their inertial motion (geodesic motion), are free bodies. Hence, the real vertically upward force on such a free body, necessary to prevent its free-fall, and acting without producing any vertically upward velocity, can obviously be only an upward centripetal force. Centripetal forces are real and act in a direction normal to the velocity vector of the moving body. It only continuously changes the direction of the body's velocity vector, letting its magnitude perfectly constant and hence, producing no velocity along its instantaneous direction. Obviously, this centripetal force can be non-null only if the orthogonal velocity of the body too is non-null. The inertial force reacting against the real (upward) centripetal force is a downward inertial or centrifugal pull, a fictitious force. If, in the reference of an observer, a body, hold stationary, acts an inertial pull, this reference cannot be an inertial reference frame. Its origin may be accelerating or it may be rotating round some axis. If this axis is not accelerated at all and the inertial pull of the body increases with the distance from the axis, it obviously is a centrifugal pull. This means that the reference of the observer is rotating and the body is stationary in it, thanks to an adequate centripetal force. For instance, in a rotating carousel, every matter body, stationary in it, is under a real centripetal force (toward the rotation axis) and exerts an outward centrifugal pull on this axis. Also a sphere on an even and smooth rotating table behaves like a free body in a rotating reference. Now on earth the fictitious gravitational force or gravitational pull on all matter bodies, stationary on it, which increases with decreasing altitude, clearly is a centrifugal pull (a fictitious force) toward the gravitational center. In this scenario, all the local earth-based laboratories are non-inertial references implicitly rotating round an over-head fixed axis under a real upward centripetal force. In order to implement correctly this inertial dynamics, without running into a stalemate, the local true inertial references must be truly rotating in the ordinary space, oppositely to the implicit rotation of the non-inertial references, round the same fixed over-head axis. The idea of a ro- 
tating reference being an inertial reference could seem stupid. If however, it is the space, ruling the inertial motion of matter-energy that is itself locally so rotating, this is not stupid at all, however a genuine "eureka". The coming Sections show that this inertial dynamics is perfectly accomplished, if such a space, is moving horizontally according to a velocity field, in which the magnitude of the velocity increases with decreasing altitude. Auspiciously, the Higgs theory introduces exactly such a real physical space, giving mass to the elementary particles by the Higgs mechanism and ruling the inertial motion of matter-energy. A non-uniform velocity field of this real Higgs Quantum Space (HQS) refracts the matter-waves analogously as wind gradients refract the sound waves in the atmosphere and thereby gives rise to an inertial dynamics, which, according to Einstein's equivalence of gravitational and inertial effects, is gravitational dynamics.

The HQS is a real quantum fluid spatial medium that gives inertial mass to the elementary particles by the Higgs mechanism and rules their inertial motion. Quantum fluids have zero viscosity, are totally frictionless and perfectly conservative. They couple only to very specific fields and are extremely difficult to notice directly. The next Section 2 gives details about the most relevant properties of the HQS. The subsequent Sections show that a Keplerian velocity field of this HQS round the sun, consistently with the planetary orbital motions $(G M / r)^{1 / 2}$ as well as round earth, consistently with the orbital motion of the Moon, accurately creates the observed gravitational dynamics in the solar system and on earth and adequately causes all the observed effects of the gravitational fields on light and on clocks. This HQS materializes the local Lorentz frames turning them into local proper Lorentz frames that are intrinsically stationary with respect to the local HQS and thus moving with it in its motions. This HQS dynamics naturally implements the inside-outside and outside-inside centrifuge scenario, generated by the gravitational fields that is the true hush-hush of the gravitational physics.

Actually, many physicists fully trust the theory of relativity (TR). However, besides the problems appointed above, the model of the free-falling inertial references of GR also has other grave shortcomings. It cannot explain the observed non-Keplerian rotation rate of the galaxies [5] [6]. The spacetime curvature, generated by the whole visible matter in the galaxies, is much too weak to hold together the rotating galaxies. Instead of suspecting the current theories, people postulate the existence of a huge amount of exotic and invisible dark matter, five times more than the whole visible hadronic matter within the galaxies. Apparently, the flawed theories are driving people to believe in phantasms! The current theories too cannot explain the origin of the observed accelerating expansion of the universe [7] [8]. This accelerating expansion requires an amount of energy (dark energy) 14 times larger than the whole visible matter-energy in the universe. According to the Glashow-Weinberg-Salam electroweak model, the electroweak breakdown has opened an energy gap of about $200 \mathrm{GeV}$ between the 
unbroken and the broken electroweak symmetry. The only known physical process that can provide such a huge amount of energy is the breakdown of the $U(1)$ symmetry of the weak force doublet immediately after the big-bang, when the temperature fell through $10^{15} \mathrm{~K}$, with condensation of the Higgs Quantum Space (HQS). The discussion about the accelerating expansion of the universe, in terms of Einstein's cosmological constant and the vacuum energy density, now is completing nearly a century. While the vacuum energy density, estimated with base in particle physics is $10^{110} \mathrm{erg} / \mathrm{cm}^{3}$, the experimentally observed value is $10^{-10} \mathrm{erg} / \mathrm{cm}^{3}$. The gap between the theoretical estimates and the experimental observations achieves 120 decimal orders of magnitude. In this scenario, a solution of the impasse is well out of sight. Therefore, the discussion about the origin of the accelerated expansion of the universe is more actual than ever. Visibly, radical changes in our view about the nature of empty space are required. Refs. [9] [10] [11] show that, in the scenario of an adiabatic universe and within the scenario of the HQS dynamics, all these problems get natural solutions.

In conclusion, GR is a mathematical model that can describe many effects of the gravitational fields. It however does not provide an acceptable physical explanation. It is an Aristotelian like explanation: Bodies and their references fall because their place is on ground. Because of Einstein's peculiar view about the nature of empty space and unknown origin of the inertial mass such a physical explanation was well out of reach. The Higgs theory has introduced the quantum fluid space and provided, by the first time, a scientifically sane explanation for the origin of the inertial mass and the inertial behavior of matter-energy. The Higgs quantum space is much more than simply a reference for rest and for motions. It literally governs the inertial motion of matter-energy and is the fundamental ingredient to really understand the origin of the gravitational dynamics.

The next Section 2 briefly discusses the relevant properties of the quantum fluids and the origin of the inertial mass. This is necessary to provide minimum understanding of the physics of the Higgs Quantum Space (HQS). The HQS gives inertial mass to the elementary particles by the Higgs mechanism and rules the inertial motion of matter-energy. Its motion gives rise to the gravitational fields. Sections 3 and 4 give a detailed mathematical solution of the gravitational dynamics in the solar system and on earth, showing that the HQS dynamics gravitational field of a spherical source is perfectly symmetric with orbital motions. Finally Section 5 describes a large number of experimental observations that all are perfectly and adequately produced by the HQS dynamics gravitational mechanism.

\section{The Origin of the Inertial Mass and the Foundations of the Gravitational Physics}

The origin of the inertial mass is an old puzzle. Newton has empirically defined 
the inertial mass of a free body as the ratio between the force $\boldsymbol{F}$ and the consequent acceleration $\boldsymbol{a}$. He also has extended this relation to the gravitational dynamics, where the mass $m$ of a free body is the ratio between the gravitational force $\left(G M m / r^{2}\right)$ and the gravitational acceleration $g(r)$. From Einstein's view, the origin of the inertial mass of a body arises from its connection with all the other masses in the universe. This however requires instantaneous action from the infinite, which opens more problems than it solves. A scientifically sane origin of the inertial mass was discovered only in the sixties of the past century with the Higgs theory.

According to the gauge theories, all the elementary particles (field quanta) are originally massless and moving at the velocity of light. The first clue that coupling of a field to a quantum condensate results in confinement and quantization of that field and mass terms for the field quanta was discovered in superconductivity by Anderson 1963 [12]. The superconducting condensate (SCC) can be described by the complex macroscopic Ginsburg-Landau order parameter (SCOP) $\Psi(r, \theta)=\psi(r) \mathrm{e}^{i \theta} \quad[13]$, where $\psi(r)$ is an amplitude and $\mathrm{e}^{\mathrm{i} \theta}$ is a phase factor. A magnetic field causes local phase displacements and phase disorder of the SCOP, elevating the energy of the SCC. Superconductors confine the magnetic field into quantized fluxons or expel it out by the Meissner effect [14], thereby lowering their energy. Gauge transformations of the SCOP, in the presence of a magnetic field, reveal mass terms for the electromagnetic field quanta. By this mechanism, photons acquire inertial mass within superconductors. However, the gauge transformations obviously do not create this mass. They only reveal it on testing the mobility of the photons. Acceleration of a photon within a superconductor involves additional local phase gradients of the superconducting order parameter, resulting in acceleration and additional motion (screening currents) of the SCC, which elevates its energy.

Spontaneous breakdown of the $U(1)$ symmetry at sufficiently low temperature, caused by the Bose-Einstein (BE) phase correlation between the wave-functions of bosons, gives rise to a negative potential energy (bonding) term, the value of which increases linearly with the condensate density $\rho=\Phi^{*} \Phi \quad$ (the potential well becomes deeper with increasing $\rho$ ). Another positive potential energy (anti-bonding) term arises from repulsive core interaction between the boson particles, that increases with the squared density $\rho^{2}$ and prevents collapse of the boson system. The effective potential $U(\rho)$ is mainly the sum of these two terms that has the form:

$$
U(\rho)=-n\left(\Phi^{*} \Phi\right)+m\left(\Phi^{*} \Phi\right)^{2}
$$

where the value of the negative coefficient $(-n)$ of the bonding term is considerably larger than the positive coefficient $(+m)$ of the anti-bonding term. Therefore the minimum of the effective potential energy occurs, not as usually for $\rho=0$, however for a finite value $\Phi^{*} \Phi=n / 2 m$, which is known as a non-zero vacuum expectation value. This effective potential is a local parameter that is 
highly homogeneous throughout the volume of the condensate. The reason is that the bosons become completely entangled, their uncertainty in momentum and energy tend to zero and the uncertainty in position and in time tend to infinity. They freely tunnel throughout the volume of the condensate.

According to the Higgs theory [15] [16], space is filled up throughout by a quantum condensate of Higgs boson particles (HC), with properties closely analogous to the superconducting condensate (SCC). Likewise the SCC confines the electromagnetic field by the Meissner effect [14] down to $10^{-7} \mathrm{~m}$, giving inertial mass to the photons within superconductors [12], the HC or Higgs Quantum Space (HQS) confines the weak and strong nuclear fields down to $10^{-19} \mathrm{~m}$, giving inertial mass to the vector bosons of the weak field as well as to all the elementary particles, porting hyper-charges by a direct or indirect Higgs mechanism [15] [16]. The HQS is an extremely powerful quantum fluid medium, ruled by the principles of quantum physics and likewise the SCC, is governed by a two component complex Ginsburg-Landau order parameter $\Phi(r, \theta)=\phi(r) \mathrm{e}^{\mathrm{i} \theta}$. However, while the SCC is stabilized by an energy gap of only about $1 \mathrm{meV}$ per particle and condenses only at very low temperature (a few tens of K), the HQS is stabilized by a huge energy gap, which, according to the Glashow-Weinberg-Salam electroweak model, [17] [18] achieves $200 \mathrm{GeV}$ and condenses at $10^{15} \mathrm{~K}$. This energy gap is $10^{14}$ times larger than that of the usual superfluids and SCC. The role of the HQS in the life of our universe is absolutely vital. Without it, no laws of motion would exist and the universe, as we know it, simply would be impossible.

In superconductors, a constant applied electromotive force (emf) causes an increasing phase displacement, developing an increasing phase gradient of the superconducting order parameter along the conductor and hence flow of the SCC at an increasing velocity, proportional to the magnitude of the phase gradient. Once excited, this current is perfectly persistent and perpetual. It can be stopped only by an opposite emf. A changing solenoidal vector potential, associated with a changing magnetic field, causes corresponding solenoidal screening currents, creating a Lorentz force field that confines the magnetic field by the Meissner effect [14] down to $10^{-7} \mathrm{~m}$ and tends to expel the magnetic field out from the superconductor. The Higgs mechanism is the perfect HQS analog of the Meissner effect. It confines the weak and strong nuclear fields down to $10^{-19}$ m. Apparently, these nuclear fields are associated with an HQS analog of the EM vector potential that causes a phase gradient on the Higgs order parameter and a velocity field of the HQS. Such phase displacements cause phase disorder and motion of the HQS according to a well-defined velocity field round the matter bodies, elevating the energy of the HQS. If this velocity field is non-uniform, it refracts the matter waves, generating an inertial dynamics and an inertial force field, which, according to the principle of equivalence is a gravitational force field.

The Higgs theory introduces fundamental changes in Einstein's view about the nature of empty space (vacuum), about the meaning of motions and about 
the nature of the gravitational fields. The HQS is much more than simply a local ultimate reference for rest and for motions. It literally governs the inertial motion of matter particles and the propagation of light (electromagnetic radiation) and hence necessarily is the local ultimate reference for rest and for motions. Several recent experimental observations, with the help of the atomic clocks in orbit, [19] [20] [21] demonstrate that the velocity of light is isotropic and constant (fixed) with respect to the local HQS and not with respect to every possible inertial reference. Velocity with respect to the local HQS and not relative velocity is the reason of all the effects of motion [22] [23] [24] [25].

If the HQS gives inertial mass to the elementary particles, it necessarily is responsible too for the gravitational fields, because it is mass that generates the gravitational fields. It is well-known that clocks stationary within a gravitational field show exactly the gravitational slowing predicted by GR. However, recently it was discovered that motion of the GPS clocks with earth round the sun cancels the gravitational time dilation by the solar field on them. From the present view of the HQS dynamics, this demonstrates that the orbiting earth is stationary with respect to the local HQS, which obviously can make a sense only if the HQS moves according to a velocity field consistent with the orbital motion of earth. Current theories explain the absence of the solar gravitational slowing on the GPS clocks in terms of the principle of equivalence. Accordingly, in the Lorentz frame of earth, earth is stationary, which is alleged to cancel the effects of the solar gravitational field. Curiously, the approaches of the HQS dynamics and of the current theories come to the same conclusion in the case of the GPS clocks in the solar field.

In the language of the present work, the Higgs Quantum Space (HQS) materializes the local Lorentz frames ( $L F s)$, turning them into local proper $L F s$, intrinsically stationary with respect to the local HQS. Only LFs, stationary with respect to the local HQS are proper LFs. On moving, the HQS carries these local proper LFs with it. If the motion of the HQS and hence of the local proper LFs is spatially homogeneous, it gives rise to practically no observable effects. However, if its motion is non-uniform, it refracts the matter waves, creating inertial dynamics on the matter particles that are moving with respect to the local HQS. This inertial dynamics, according to the principle of equivalence, is gravitational dynamics. References [22] [23] [24] [25] show that motion of the HQS, round the sun, according to a Keplerian velocity field, consistent with the planetary motions, accurately creates the observed gravitational dynamics and all the observed effects of the gravitational fields on light and on clocks. In terms of spherical coordinates this Keplerian velocity field for a spherical source has the very simple form:

$$
\boldsymbol{V}(r)=(G M / r)^{1 / 2} \boldsymbol{e}_{\phi}
$$

This is a cylindrical velocity field round the $Z$ axis $(\theta=0)$, in which the magnitude of the velocity of the HQS is spherically symmetric, $G$ is the gravitational constant, $M$ is the mass of the gravitational source (sun), $r$ is the radial spherical 
coordinate and $\boldsymbol{e}_{\phi}$ is a unit vector along the azimuthal spherical coordinate $\phi$.

The present work associates the central idea of GR, according to which the gravitational pull is an inertial pull and the central idea of the Higgs theory, according to which the HQS gives mass and governs the inertial motion of matter-energy and replaces Einstein's spacetime curvature by a Keplerian velocity field of the HQS. This Keplerian velocity field of the HQS is the quintessence of the gravitational fields. Within this velocity field, the observed gravitational dynamics, the gravitational pull, the absence of the solar gravitational slowing of the GPS clocks as well as the absence of the light anisotropy with respect to earth are all genuine and natural outcomes of this Keplerian velocity field of the HQS. These observations are the authentic signature of the true physical mechanism of gravity in action.

Now coming back to Equation (3), it is important to note that, in the scenario of the HQS, the electromagnetic field is not confined and the velocity $c$ of light is fixed and isotropic, not with respect every inertial reference, however with respect to the local HQS. In this scenario, the velocity of light in free space (vacuum) is the characteristic velocity at which the HQS transmits the local phase perturbations of the Higgs order parameter. The local phase perturbations and the consequent velocity field of the HQS, caused by the elementary particle fields (vector bosons, quarks and leptons), is of a quantum mechanic nature. They are confined by the Higgs mechanism down to $10^{-19} \mathrm{~m}$ and quantized according to well defined rules. However, the electromagnetic field does not couple and is not confined by the HQS. Photons have no rest mass and propagate at the characteristic propagation velocity $c$ of the HQS and not with respect to any possible inertial reference as postulated in the theory of relativity. Any local reference, stationary with respect to the local HQS is a local proper Lorentz frame (LF) with respect to which the velocity of light is fixed and isotropic. All references moving with respect to the local HQS are non-proper LFs. However, within the gravitational fields, the HQS itself is moving according to the Keplerian velocity field Equation (6). In this motion, the HQS carries the local proper inertial references (local proper LFs) with it. Therefore, within the gravitational fields, the local velocity vector of the HQS $\boldsymbol{V}=(G M / r)^{1 / 2} \boldsymbol{e}_{\phi}$ adds up to the fixed velocity vector $\boldsymbol{c}$ of light with respect to the local HQS. The effective velocity of light $c^{\prime}$ is $\boldsymbol{c}^{\prime}=\boldsymbol{c}+\boldsymbol{V}$.

Elementary particles are conceived as confined and quantized stationary wave mechanisms (particle fields), propagating locally, in a very small region of space, at the velocity of light and described by quantum mechanical wave functions. This internal dynamics is implicit and cannot be conceived in terms of classical motions in the ordinary space. Einstein has conceived this confined internal dynamics of the elementary particles, governing their time evolution, in terms of motions at the velocity of light along a fourth dimension, implicit or imaginary velocity along the time axis. However, these confined and quantized wave mechanisms can themselves move (propagate) in the form of stable particles with respect to the local HQS (ordinary motions), independently from their internal 
dynamics. However, due to their internal quantum wave nature (go-return round-trip propagation), such ordinary motions slow down the internal time evolution of the particles, as shown by atomic clocks, atoms and elementary particles.

Clocks are devices that count time in terms of a time standard, which can be a classical or quantum oscillator. The time standard of atomic clocks is the period of electromagnetic (EM) oscillations in an EM cavity, tuned to the well-defined frequency of the hyperfine transition of Cs atoms. Such clocks measure the time evolution down to tenth of nanoseconds or even better and are of course affected by velocity with respect to the local HQS (not by relative velocity). They however are slowed differently for transverse or longitudinal directions of the oscillations of the time standard with respect to this ordinary motion.

What is absolutely extraordinary from Einstein is having perceived that the confined and quantized dynamics, involved in the rest energy $E_{0}$ of the particles and the additional non-confined and non-quantized dynamics (kinetic energy $K$ ) are totally independent from each other and behave as orthogonal degrees of freedom. This will say that the total energy $E$ is obtained by a Pythagoras like formula:

$$
E^{2}=E_{0}^{2}+K^{2}=m_{0}^{2} c^{4}+p^{2} c^{2}
$$

This is Einstein's famous equation for the total energy of a moving elementary particle with rest mass $m_{0}$.

Within gravitational fields, the coefficient of the last term, in Equation (3), is the square of the effective velocity $c^{\prime}$ along the time axis (time evolution), where $c$ and $2 G \vec{M} / r$ are intrinsically independent from each other (orthogonal). Therefore, this effective velocity can be obtained by the formula of Pythagoras $c^{\prime}=\left(c^{2}\left(1-2 U / c^{2}\right)\right)^{1 / 2}=\left(c^{2}-2 G M / r\right)^{1 / 2}$. Note that, to first order, the gravitational slowing is $t_{0}(G M / r) / c^{2}$, which is well observed on clocks stationary in the earth's gravitational fields. Einstein has described this gravitational slowing of clocks in terms of the square of the escape velocity $2 U / c^{2}$. However, from the viewpoint of the present work, the gravitational time dilation of clocks, stationary in the gravitational field, created by the Keplerian velocity field Equation (6) is due to the implicit velocity $\left(\boldsymbol{v}_{\text {impl }}(r)=-(G M / r)^{1 / 2} \boldsymbol{e}_{\phi}\right)$ with respect to the local moving HQS. Moreover, in the scenario of the HQS, the period for longitudinal electromagnetic oscillations $\left(t_{l}=t_{0}\left(1-(G M / r) / c^{2}\right)^{-1}\right)$ of the stationary clock's time standard is lengthened by $\approx t_{0}(G M / r) / c^{2}$, while for transverse roundtrips $\left(t_{t}=t_{0}\left(1-G M / r / c^{2}\right)^{-1 / 2}\right)$ it is lengthened lesser by $\approx(1 / 2) t_{0}(G M / r) / c^{2}$. Due to his view about motions in a four-dimensional empty spacetime, Einstein never has considered the possibility of longitudinal light round-trips. Depending on the direction of observation with respect to the direction of the electromagnetic oscillation in the sources, emitting dipole radiation, it can be selective for longitudinal or transverse oscillations. Photons emitted along the motion are selective for transverse EM oscillations.

From the viewpoint of the present work, in free space (zero gravitational field), 
the effective velocity $c^{\prime}$ along the time axis can be obtained by the formula of Pythagoras $c^{\prime}=\left(c^{2}-v^{2}\right)^{1 / 2}$, where $c$ is the fixed velocity of propagation in the local HQS (vacuum) and $v$ is the ordinary velocity of the clock, the velocity of the laboratory with respect to the local stationary HQS. However, within a gravitational field, the HQS is itself moving according to the Keplerian velocity field Equation (6) along $+\phi$. In this case the local effective velocity along the time axis of a clock, stationary in the gravitational field too can be calculated by the formula of Pythagoras $c^{\prime}=\left(c^{2}-G M / r\right)^{1 / 2}$, where $c$ again is the fixed velocity, the parameter of evolution of the confined phase perturbations (particles) with respect to the local now moving HQS. However, $G M / r$ is the square of the orthogonal implicit velocity of the stationary particle (clock) along $-\phi$ within the Keplerian velocity field, creating the gravitational field. For longitudinal oscillations of the clock's time standard, the rate of the clock is $t_{l}=t_{0}\left(1-(G M / r) / c^{2}\right)^{-1}$. To first order, this gives a clock slowing of $\approx t_{0}(G M / r) / c^{2}$, which is the same as in GR.

Now, the first $(r)$ term in Equation (3) describes stretching of the radial distances, due to the gravitational field $\mathrm{d} r^{2}=\mathrm{d} r_{0}^{2} /\left(1-2 U / c^{2}\right)$. According to GR, this stretching, from the view of an external observer, causes an apparent reduction of the velocity of light, propagating toward the gravitational center. However, from the view of the present HQS dynamics, the velocity of a light beam, propagating toward the gravitational center, decreases really and not apparently. It decreases because it necessarily develops an implicit orthogonal velocity component $(G M / r)^{1 / 2}$ along $-\phi$. The time interval, necessary for light to propagate a distance $\mathrm{d} r$ along the radial coordinate, is $\mathrm{d} t=\mathrm{d} r / c=\mathrm{d} r_{0} /\left(c^{2}-G M / r\right)^{1 / 2}$, where $\left(c^{2}-G M / r\right)^{1 / 2}$ is again the effective velocity $c^{\prime}$ of light, obtained by the formula of Pythagoras. However this $c^{\prime}$ is only the radial velocity component of light. The dilation of the radial distances in GR is consequence of postulating the implicit constancy of the velocity of light, which scales the radial distances in units of the effective radial velocity of light.

In the Keplerian velocity field of the sun, earth is very closely stationary with respect to the local moving HQS as well as with respect to the local proper Lorentz frames. Moreover, the solar system too is very closely stationary with respect to the local moving HQS and moving closely within the equatorial plane in the HQS velocity field of our Milky-Way galaxy etc. This cancels the solar gravitational pull on earth, because a gravitational pull can only be generated on a matter body if it has a non-null velocity with respect to the local HQS. It also cancels the gravitational slowing of the GPS clocks by the solar field, cancels the light anisotropy with respect to earth and cancels all the other effects of the solar gravitational field on light and on clocks moving together with the orbiting earth. Visibly, the astronomical bodies throughout the universe are all very closely stationary with respect to the local HQS in the respective gravitational fields, which automatically leads to the universality of the laws of physics and needs not the Principle of Relativity. 
The macroscopic quantum physics of the HQS plays a fundamental role in the physics of the microscopic quantum world (giving mass and governing the inertial motion of the elementary particles) as well as in the physics in the macroscopic world of gravitation (macroscopic inertial dynamics). The HQS thus is the natural link between quantum physics and gravitation. It links together the physics of particles, of stars, of galaxies and integrates the universe into a wholly coherent system.

Clocks, not orbiting in the equatorial plane of the Keplerian velocity field, must have considerable velocity with respect to the local HQS. A clock, moving in a direct circular equatorial orbit, is predicted to show proper time. However, a clock, moving in a polar orbit, runs two times slower than a clock stationary at the same altitude and a clock in a retrograde circular equatorial orbit runs four time slower than a clock at the same altitude. In particular, the orbital motions of the GPS clocks round earth make 55 degrees with respect to the earth's equator. They have a Westward velocity component of $3.87(1-\cos \alpha) \mathrm{km} / \mathrm{sec}$ with respect to the local HQS (with respect to the local proper LFs) and also a North-South velocity component of $3.87 \sin \alpha \mathrm{km} / \mathrm{sec}$, where $\alpha$ is the angle of the orbital velocity with the equator or parallels. The resultant velocity of the satellites with respect to the local HQS, in the equatorial region, is given by $3.87[2(1-\cos \alpha)]^{1 / 2}=3.574 \mathrm{~km} / \mathrm{sec}$ along the most part of the orbit. The average velocity along the whole orbit is approximately $2.86 \mathrm{~km} / \mathrm{sec}$. Analogously the Westward velocity of the earth-based station at Colorado highs with respect to the local HQS is about $7.4 \mathrm{~km} / \mathrm{sec}$. To these velocities of the satellites and of the ground based clock we must consider the velocity of the Cs atoms of 0.255 $\mathrm{km} / \mathrm{sec}$ within the atomic clocks and on the clocks on earth as well as a small transverse Doppler shift, due to the implicit velocity of the earth-based stations with respect to the local HQS. Altogether these effects achieve closely the observed $4.5 \times 10^{-10} \mathrm{sec} / \mathrm{sec}$.

In the Keplerian velocity field of the HQS, the local inertial references (IRs) are not free-falling, however rotating, in the ordinary space, round a fixed over-head axis. The axis of an IR located at $\left(r_{0}, \theta_{0}, \phi_{0}\right)$, lies at $\left(2 r_{0}, \theta_{0}, \phi_{0}\right)$ and points along the $+\theta$ coordinate. In order to rotate round such fixed over-head axes, the point $\left(r_{0}, \theta_{0}, \phi_{0}\right)$ of the local IR needs to move along the $+\phi$ coordinate together with the local HQS and the local proper LFs at a velocity given by Equation (6) round the gravitational center. The fact that, in the Keplerian velocity field, the velocity of the HQS and of the local LFs increases with decreasing radial coordinate, has the consequence that the distribution of velocity, through any infinitesimal region within the $[r, \theta]$ plane, corresponds to a rotation round the overhead axis. The local angular velocity is given by $W=+\left((G M / r)^{1 / 2}\right) / r \boldsymbol{e}_{\theta} \mathrm{rad} / \mathrm{sec}$, where $\boldsymbol{e}_{\theta}$ is a unit vector along the $+\theta$ spherical coordinate. In the static situation (body stationary in the ordinary space), this rotation is the active principle responsible for the gravitational pull.

Einstein could have concluded from the null light anisotropy results that earth 
is stationary with respect to the local space ruling the motion of matter and the propagation of light and that consequently this space is moving round the sun, consistently with the planetary motions. However, instead of this, probably due to his view about nature of empty space and the consequent lack of a local ultimate reference for rest and for motion, Einstein, has postulated the intrinsic isotropy and constancy of the velocity of light. This postulate has closed the possibility of understanding gravity straightforwardly as a conventional inertial dynamics.

The Keplerian velocity field of the HQS is the quintessence of the gravitational fields. It is the only possible velocity field of the HQS, ruling the inertial motion of matter-energy that is able to create the outside-inside and inside-outside centrifuge phenomenon, observed within the gravitational fields. This unconventional inertial dynamics is the true hush-hush of the gravitational fields that is so difficult to understand. The corresponding observed centrifugal pull, exerted by matter bodies that are somehow impeded to follow their inertial motion, is a central field of usual inertial (centrifugal) forces (fictitious forces) toward the gravitational center that Newton has mistakenly seen as the central field of real gravitational forces.

\section{The Gravitational Dynamics in the Solar System and on Earth}

The Keplerian velocity field of the HQS (Equation (6)), consistent with the planetary motions and creating the solar gravitational field achieves $436 \mathrm{~km} / \mathrm{sec}$ on the solar surface. The solar Keplerian velocity field carries the planets with it along direct circular equatorial orbits, while they are very closely stationary with respect to the local moving HQS and also with respect to the local proper LFs. This is their local resting condition. Motion along circular equatorial orbits cancels locally all the effects of the gravitational field on matter, on light and on clocks. Therefore, light is closely isotropic with respect to earth and also with respect to the other planets of the solar system. Moreover, clocks, moving with these planets, display very closely proper time. According to the observations, the velocity of earth with respect to the local HQS in the solar field is only of a few hundreds of meters per second, the effect of which on light and on clocks is too low to be detected even by the current most sensitive instruments. However, the refraction rate of this small velocity is sufficient to make the orbits of the planets a little bit elliptic. Please see ref. [9] and also refs. [22] [23] [24] [25] for the full details.

The velocity of the HQS in the Keplerian velocity field (Equation (6)) round earth, in the sense of the Moon's orbital motion (from West to East) and creating the earth's gravitational field, achieves $7.91 \mathrm{~km} / \mathrm{sec}$ on surface. However, as earth rotates toward the East only very slowly ( $465 \mathrm{~m} / \mathrm{sec}$ at the equator), the earth-based laboratories are moving at an implicit velocity toward the West at velocities, ranging from $7.445 \mathrm{~km} / \mathrm{sec}$ at the equator up to $7.91 \mathrm{~km} / \mathrm{sec}$ at the 
poles. This velocity creates the gravitational acceleration and the gravitational pull of matter bodies on earth. It also creates the very small light anisotropy of nearly $8 \mathrm{~km} / \mathrm{sec}$, barely detectable by the most sensitive Michelson light anisotropy experiments [26] (please see Section 5.2 hereafter) and the very small gravitational slowing of the atomic clocks and the spectral red-shifts, observed on earth. However, although very small, these effects of the earth's field are much more relevant than the undetectably small gravitational slowing of the GPS clocks and the light anisotropy effects, caused by the solar field on earth. These last simply seem to be absent.

The rotation rate (refraction) of the velocity components of a particle in the Keplerian velocity field is not a trigonometric rotation. In fact the Keplerian velocity field is intermediary between rigid body rotation and potential flow with zero curl. The local velocity distribution of the HQS in the earth's Keplerian velocity field through the $[r, \theta]$ plane, seen by a particle with a velocity along the $\phi$ direction $\left(v_{\phi}\right)$ and its de Broglie wave fronts in the $[r, \theta]$ plane, corresponds to rotation of the HQS and of the local inertial reference (IR) round an overhead axis. However, the wave-fronts in the $[\theta, \phi]$ plane of the radial velocity component $-v_{r}(t)$ do not see this rotation rate round the overhead axis. They see the opposite rotation of the HQS round the gravitational center. The $\theta$ velocity component is not refracted at all, because the velocity field Equation (6) has no component along $\theta$. Besides these refraction rates, there is need of considering the wavelength stretching and shortening, due to the deformations of the HQS in the Keplerian velocity field, that affect the velocity of the particles according to de Broglie's equation $p=h / \lambda$. However the $r$ and the $\phi$ velocity components are not affected by these wavelength deformations. Hence, describing the effects of the gravitational field, in terms of these velocity components and their effective rotation rates, the effects of wavelength stretching-compression are automatically taken into account.

In order to obtain quantitative values for the rotation rates of the $r$ and $\phi$ velocity components, the orbital velocity $v_{\text {orb }}$ of a particle in an elliptic orbit $(\epsilon=0.5)$ was very precisely computed and plotted in Figure 1 together with the Keplerian velocity $V(r)$, given by Equation (6) and the effective velocity with respect to the local HQS, at a large number of points along the orbit, taking into account the relation: $v_{\text {eff }}=\boldsymbol{V}(r)+\boldsymbol{v}_{\text {orb }}$.

The obtained refraction rates of the $-r,-\phi$ and the $\pm \theta$ velocity components are:

$$
\begin{gathered}
\boldsymbol{W}_{r}(r)=-\frac{1}{2}\left[G M / r^{3}\right]^{1 / 2} \boldsymbol{e}_{\theta} \\
\boldsymbol{W}_{\phi}(r)=+\left[G M / r^{3}\right]^{1 / 2} \boldsymbol{e}_{\theta} \\
\boldsymbol{W}_{\theta}(r)=0
\end{gathered}
$$

Note the opposite signs in the rotation rate of the $-r$ velocity component and 


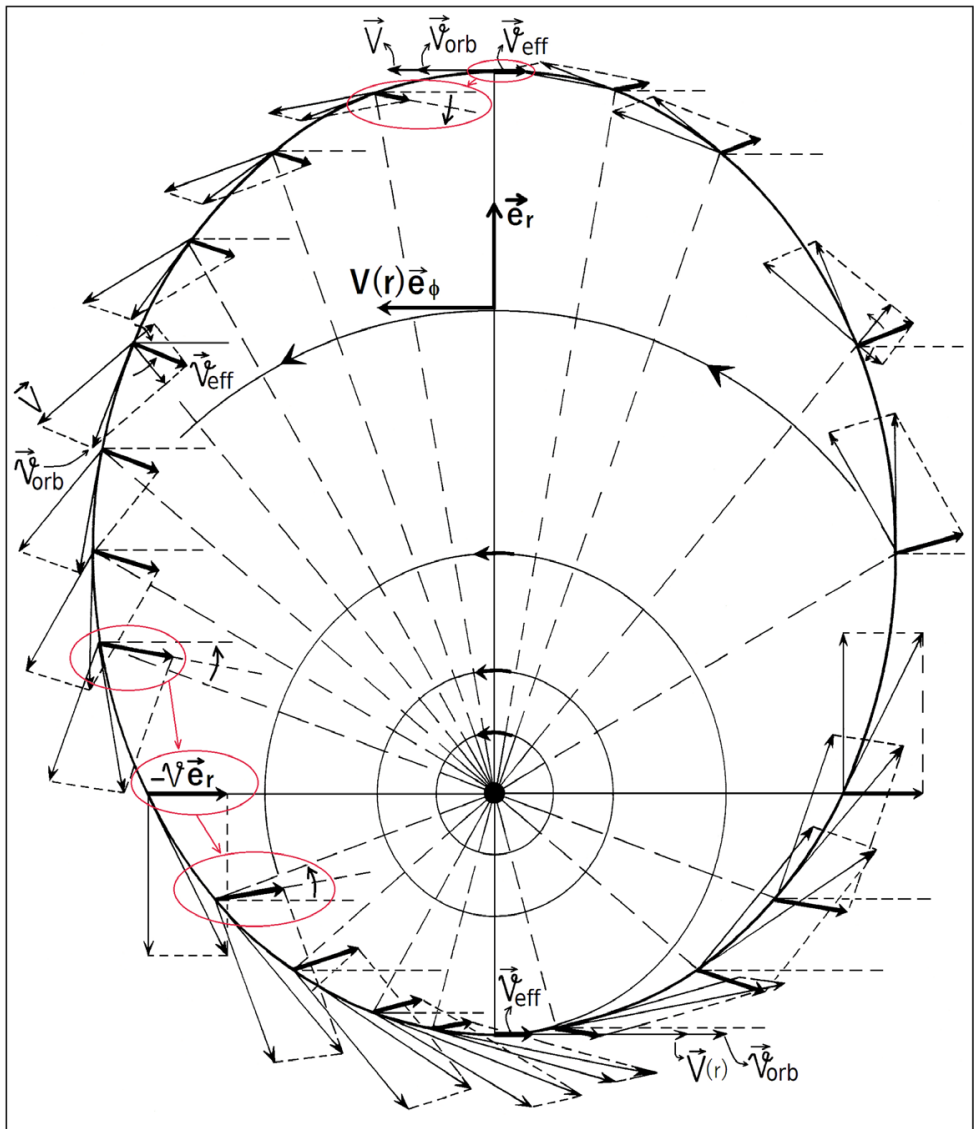

Figure 1. The figure is a very precise graphical representation. The velocity diagrams are shown at a large number of positions along the elliptic orbit, where $\boldsymbol{v}_{\text {eff }}$ is represented by the bulky arrows, pointing toward the right. At the top, the rotation rate of the effective $-\phi$ velocity component can be read. The rotation rate of the $-r$ velocity component can be read at the left. Please see the encircled diagrams.

the $-\phi$ velocity component.

The implicit velocity (along $-\phi$ ) of a particle stationary in the gravitational field is given by:

$$
\boldsymbol{V}_{\text {impl }}=-(G M / r)^{1 / 2} \boldsymbol{e}_{\phi}
$$

The refraction rate of this implicit velocity, according to Equation (8b), generates an ordinary vertical downward acceleration given by:

$$
\boldsymbol{g}(t)=W_{\phi}(r) \boldsymbol{e}_{\theta} \times V_{\text {impl }}(r)\left(-\boldsymbol{e}_{\phi}\right)=-G M / r^{2} \boldsymbol{e}_{r}
$$

This is the usual expression for centrifugal accelerations in a rotating reference. Note that here, the particle, stationary at a generic point of the ordinary space, in fact is stationary with respect to a non-inertial reference that is implicitly rotating oppositely with respect to the local truly rotating inertial reference in the ordinary space, according to Equation (8b). Here $\boldsymbol{g}(r)$ has clearly the nature of a centrifugal acceleration, pointing from everywhere to the gravitational center. $\boldsymbol{g}(r)$ is a spherically symmetric field of centrifugal accelerations 
toward the gravitational center. A body, hold fixed with respect to the ordinary space coordinates (non-inertial earth-based laboratory) within a gravitational field, necessarily will implicitly be moving along a circular path within the local truly rotating IR, round the same overhead axis, under a Real upward centripetal force.

Equation (10) is sufficiently precise only for free-fall along short distances. For large distances of free-fall, the following elementary linear differential equation must be solved:

$$
\frac{\mathrm{d} \boldsymbol{v}(r(t))}{\mathrm{d} t}=\boldsymbol{A} \boldsymbol{v}_{0}
$$

where $\boldsymbol{v}$, is the column matrix of the (ordinary) $r$ and the (implicit) $\phi$ velocity components of the effective velocity with respect to the local HQS:

$$
\boldsymbol{v}(t)=\left(\begin{array}{l}
v_{r}(t) \\
v_{\phi}(t)
\end{array}\right)
$$

In Equation (11), $\boldsymbol{A}$ is the hyperbolic infinitesimal rotation matrix along parallel axes, defined in terms of the rotation rates, given in Equation (8):

$$
\boldsymbol{A}=\left(\begin{array}{cc}
0 & W_{\phi} \mathrm{d} t \\
-W_{r} \mathrm{~d} t & 0
\end{array}\right)=\left(\begin{array}{cc}
0 & W \mathrm{~d} t \\
\frac{1}{u} W \mathrm{~d} t & 0
\end{array}\right)
$$

where $W=G M / r^{3}$ and $u=2 M /(M+m)$ accounts for the asymmetric distribution of kinetic energies between the interacting masses $m$ and $M$.

Dividing both sides of Equation (11) by $\boldsymbol{v}_{0}$, multiplying them by $\mathrm{d} t$ and integrating the left hand side from $\boldsymbol{v}_{0}$ to $\boldsymbol{v}$, develops into:

$$
\log \frac{\boldsymbol{v}(t)}{\boldsymbol{v}_{0}}=\int_{0}^{t} A\left(r\left(t^{\prime}\right)\right) \mathrm{d} t^{\prime}
$$

which can be rewritten in the exponential form as:

$$
\boldsymbol{v}(t)=\boldsymbol{v}_{0} \exp \left[\int_{0}^{t} A\left(r\left(t^{\prime}\right)\right) \mathrm{d} t^{\prime}\right]
$$

Expanding the exponential in series and adding up the terms of the series from $n=0$ to $n=\infty$ results in:

$$
\begin{aligned}
\boldsymbol{v}(t) & =\sum_{n=0}^{\infty} \frac{1}{n !}\left(\begin{array}{cc}
0 & \Theta(t) \\
\frac{\Theta(t)}{u} & 0
\end{array}\right)^{n}\left(\begin{array}{c}
v_{r}(0) \\
v_{\phi}(0)
\end{array}\right) \\
& =\left(\begin{array}{cc}
\cosh \left(\frac{\Theta(t)}{\sqrt{u}}\right) & \sqrt{u} \sinh \left(\frac{\Theta(t)}{\sqrt{u}}\right) \\
\frac{1}{\sqrt{u}} \sinh \left(\frac{\Theta(t)}{\sqrt{u}}\right) & \cosh \left(\frac{\Theta(t)}{\sqrt{u}}\right)
\end{array}\right) \times\left(\begin{array}{l}
v_{r}(0) \\
v_{\phi}(0)
\end{array}\right)
\end{aligned}
$$

The value of $\Theta(t)$ can be computed by integration: 


$$
\begin{aligned}
\Theta(t) & =\int_{0}^{t} W\left[r\left(t^{\prime}\right)\right] \mathrm{d} t^{\prime} \\
& =\int_{r_{0} \mathrm{cM}}^{r^{\mathrm{CM}}}\left[\frac{G M}{\left(r^{\mathrm{CM}}+R^{\mathrm{CM}}\right)^{3}}\right]^{1 / 2} \frac{\mathrm{d} r^{\mathrm{CM}}}{\dot{r}^{\mathrm{CM}}} \\
& =-\sqrt{u} \cosh ^{-1}\left[\frac{r_{0}^{\mathrm{CM}}}{r^{\mathrm{CM}}(t)}\right]^{1 / 2}
\end{aligned}
$$

Inversion of the final term of Equation (17) results in expressions for the hyperbolic cosines and sines:

$$
\begin{gathered}
\cosh \left(\frac{\Theta(t)}{\sqrt{u}}\right)=\sqrt{\frac{r_{0}^{\mathrm{CM}}}{r^{\mathrm{CM}}}}=\sqrt{\frac{r_{0}}{r}} \\
\sinh \left(\frac{\Theta(t)}{\sqrt{u}}\right)=\sqrt{\frac{r_{0}^{\mathrm{CM}}-r^{\mathrm{CM}}}{r^{\mathrm{CM}}}}=\sqrt{\frac{r_{0}-r}{r}}
\end{gathered}
$$

where the last equality is obvious. Using this result and noting that $v_{\phi}^{\mathrm{CM}}=v_{\phi}$, Equation (16) becomes:

$$
\left(\begin{array}{c}
v_{r}(t) \\
-v_{\phi}(t)
\end{array}\right)=\left(\begin{array}{l}
\sqrt{r_{0} / r} \sqrt{u\left(r_{0}-r\right) / r} \\
\sqrt{\left(r_{0}-r\right) / u r} \sqrt{r_{0} / r}
\end{array}\right) \times\left(\begin{array}{c}
v_{r}(0) \\
-v_{\phi}(0)
\end{array}\right)
$$

For free fall of $m$ in the field of $M(m \ll M)$, on from $r_{0}$ and initial rest, where $v_{r}(t=0)=0$ and $\boldsymbol{v}_{\phi}(0)=\boldsymbol{V}_{\text {impl }}\left(r_{0}\right)=-\left(G M / r_{0}\right)^{1 / 2} \boldsymbol{e}_{\phi}$, the final solution of Equation (11) is:

$$
\begin{gathered}
v_{r}(t)=\left[2\left(\frac{G M}{r(t)}-\frac{G M}{r_{0}}\right)\right]^{1 / 2} \\
-v_{\phi}(t)=-V_{\text {impl }}\left(r_{0}\right)\left[\frac{r_{0}}{r}\right]^{1 / 2}=-\left[\frac{G M}{r(t)}\right]^{1 / 2}
\end{gathered}
$$

Equation (20a) is just the well known expression for the observed vertical free-fall on from rest at $r_{0}$, directly showing that the kinetic energy is equal to the variation of the potential energy. Equation (20b) is just the implicit (imaginary) velocity as a function of the radial position $r$. This shows that the refraction rate of the radial velocity component just compensates for the increase of the velocity field as a function of the decrease of the radial coordinate. This assures that free-fall of the particle, on from rest, goes along a vertical (radial) path and hence assures conservation of the angular momentum about the gravitational center. Please observe that, for free-fall on from infinity $\left(r_{0}=\infty\right)$, the vertical velocity $v_{r}(r(t))$ is exactly $\sqrt{2}$ times larger than the $\phi$ velocity $v_{\text {phi }}(r(t))$, exactly as observed, accomplishing the Virial theorem.

\section{Symmetry of the Gravitational Dynamics with Orbital Motions}

Consider free-fall experiments at the equatorial region of a rotating planet like 
earth. The effective velocity $v_{\text {eff }}$ of a particle, initially stationary near to the surface of a planet, rotating with an angular velocity $\omega$ in the same sense or the opposite sense round the same axis as the Keplerian velocity field, will be given by:

$$
\boldsymbol{v}_{\text {eff }}(\theta)=\boldsymbol{V}_{\text {impl }}(R)+\boldsymbol{v}_{\text {rot }}(\theta)=-\left[(G M / R)^{1 / 2} \mp \omega R \sin \theta\right] \boldsymbol{e}_{\phi}
$$

where $\boldsymbol{v}_{\text {rot }}(\theta)$ is the ordinary velocity, due to the planet's rotation that depends on the latitude via $\sin \theta$. The upper and lower signs are respectively for direct and or retrograde rotation of the planet with respect to that of the local HQS.

However, rotation of the planet in the same sense as the Keplerian velocity field of the HQS, gives rise to a trigonometric rotation rate of the effective velocity $v_{\text {eff }}$ of the particle, due to the planets rotation that adds up to the refraction rate, given by Equation ( $8 \mathrm{~b})$. However, for retrograde rotation, it subtracts, so that the effective rotation rate of the effective velocity vector is:

$$
\boldsymbol{\omega}_{\text {eff }}(\theta)=\frac{1}{R}\left[V_{\text {impl }} \pm\left(v_{\text {rot }}\right)\right] \boldsymbol{e}_{\theta}=\left[\left(G M / R^{3}\right)^{1 / 2} \pm \omega \sin \theta\right] \boldsymbol{e}_{\theta}
$$

where the same convention for the upper and the lower signs as in Equation (21) is used.

Considering the effective velocity (Equation (21)) and the effective rotation rate (Equation (22)), the effective gravitational acceleration on the planet's surface is:

$$
\boldsymbol{g}_{\text {eff }}(\theta)=\omega_{\text {eff }} \times \boldsymbol{v}_{\text {eff }}=-\left[G M / R^{2}-\omega^{2} R \sin ^{2} \theta\right] \boldsymbol{e}_{r}
$$

The first term in the right hand side of Equation (23) describes the gravitational acceleration toward the gravitational center of the planet in the static situation (see Equation (10)), while the second term is an outward centrifugal term. Equation (23) shows that the effective gravitational acceleration $\boldsymbol{g}_{\text {eff }}(\theta)$ on the rotating planet's surface is perfectly symmetric for direct or retrograde rotation of the planet and thus also for direct or retrograde orbital motion of the particle.

For strictly circular polar orbits with radius $r>R, \boldsymbol{v}_{\text {eff }}$ has velocity components along $-\phi$ as well as along $\theta$. The velocity along $-\phi$ is:

$$
\boldsymbol{v}_{\phi}=\boldsymbol{V}_{\text {imp }}(r)=-[G M / r]^{1 / 2} \boldsymbol{e}_{\phi}
$$

Along theta the velocity is:

$$
\boldsymbol{v}_{\theta}= \pm[G M / r]^{1 / 2} \boldsymbol{e}_{\theta}
$$

While $\boldsymbol{v}_{\phi}$ generates the gravitational acceleration $\boldsymbol{g}(r)=-G M / r^{2} \boldsymbol{e}_{r}$, see Equation (10), the $\theta$ velocity component is not affected directly by the HQS-dynamics, because the velocity field Equation (6) has no velocity component along theta. However, the rotation rate of the $\phi$ velocity component plays the role of a centripetal acceleration toward the gravitational center that bents the $\theta$ velocity toward the earth's surface. The effective gravitational acceleration 
for polar orbits is:

$$
\boldsymbol{g}_{\text {eff }}(r)=-\left[G M / r^{2}-\left(v_{\theta}^{2}\right) / r\right] \boldsymbol{e}_{r}
$$

where again the first term in the right hand side is the acceleration toward the gravitational center (please see Equation (10)), while the second term is the corresponding usual upward centrifugal effect.

Together the results, expressed by Equations (23) and (26), show that the effects of the HQS dynamics and the effects of the ordinary motion within the gravitational field are completely independent. They are orthogonal. While the Keplerian velocity field of the HQS (Equation (6)) simulates a central field of fictitious Newtonian gravitational forces, the ordinary orbital motions generate the centrifugal effects, exactly as conceived in Newtonian gravity. This shows that treating the motions within a spherically symmetric gravitational field as motions in a hypothetical inertial reference, extending over the whole region, under a hypothetical central field of fictitious gravitational forces although not corresponding to reality, gives closely the observed gravitational dynamics. This explains why the Newtonian gravitational theory, although based in the fictitious gravitational forces, works so well.

\section{Effects of the HQS Dynamics Gravitational Mechanism on Light and on Clocks}

The purpose of this Section is showing that the Keplerian velocity field of the HQS naturally and correctly predicts all the observed effects of the gravitational fields on light and on the rate of clocks.

\subsection{Precise Measurements of the One-Way Light Velocity between Artificial Satellites}

In order to measure the one-way velocity of light, it is necessary to have well synchronized clocks at each end of the travel distance. From the viewpoint of the present HQS dynamics gravitational mechanism, synchronization of orbiting clocks by Einstein's method can be very precise, if the signal path between the earth-based station and the satellite is perpendicular to the local Keplerian velocity field of earth. This is the case when the satellite is vertically above the station. In the case of polar orbits, this is especially favorable, because the signal path is almost perpendicular to the HQS velocity along the whole orbit.

Actually the one-way velocity of light can precisely be measured with the help of the tightly synchronized atomic clocks in orbit to within $0.1 \mathrm{~ns}$, time for light to travel $3 \mathrm{~cm}$. The most precise measurements were achieved with the help of atomic clocks in the robotic twin satellites of the GRACE project, moving both in the same polar orbit and separated by $\sim 200 \mathrm{~km}$. In order to measure the micro-gravity effects, these clocks need to be synchronized to better than $0.16 \mathrm{~ns}$. Continuous exchange of EM signals between these satellites, in both senses, has revealed a clear anisotropy of the signal velocity of nearly $8 \mathrm{~km} / \mathrm{sec}(\sim 17 \mathrm{~ns})$ 
backward to the motion of the satellites [21]. This anisotropy is equal to the orbital velocity of these satellites. As the velocity field Equation (6) has no component along polar orbits, this observation is reliable and provides experimental evidence that a spatial medium (HQS) exists, propagating the EM signals (light) at a well defined fixed velocity $c$. The immediate consequence of this observation is the need of finding a new interpretation for all the light anisotropy experiments performed in the past century (please see next Section 5.2).

\subsection{The Michelson Light Anisotropy Experiments}

The large majority of the Michelson experiments, searched for light anisotropy due to the orbital and cosmic motion of earth. They all found closely null results. According to the present work, this confirms that earth is very closely stationary with respect to the local HQS. Earth is moving round the sun along a direct circular equatorial orbit together with the local HQS in the solar Keplerian velocity field. These null results also show that the solar system is stationary in the velocity field of the HQS, generating the gravitational dynamics of the Milky-Way galaxy. The null light anisotropy results moreover show that the accelerated expansion of the universe is concomitant with the expansion of the HQS itself.

In the view of the present work, the only motion, that causes relevant anisotropy of light with respect to the earth-based laboratories, is the local velocity field of the HQS round earth itself in the sense of the Moon's orbital motion (West-East), reaching $7.91 \mathrm{~km} / \mathrm{sec}$ on the earth-surface and creating the earth's gravitational field. The predicted light anisotropy is only of nearly $8 \mathrm{~km} / \mathrm{sec}$, constant the whole day and the whole year and thus extremely difficult to detect. The Michelson light anisotropy experiments with the highest sensitivity and rotating within the earth-based laboratories found nearly West East anisotropies of nearly $8 \mathrm{~km} / \mathrm{sec}$, constant the whole day and the whole year [26], exactly as predicted here. Figure 2 displays Miller's results.

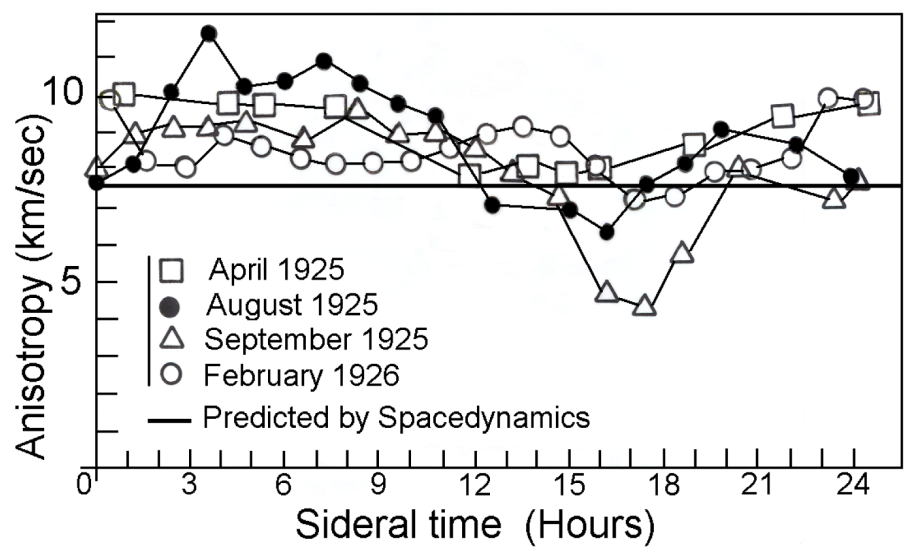

Figure 2. The Nearly West-East light anisotropy, with respect to the earth based laboratories, obtained 1925 and 1926 and nearly constant the whole day and the whole year, by D. Miller. 


\subsection{Gravitational Time Dilation and Gravitational Spectral Red Shifts}

The gravitational spectral red-shifts of atoms and the gravitational time dilation of clocks, stationary in the gravitational field are now well confirmed experimentally [3] [27] [28]. While in GR these effects are calculated like transverse light round-trip effects $\left(t(r)=t_{0}\left(1-(2 G M / r) / c^{2}\right)^{-1 / 2}\right)$, where $2 G M / r$ is the square of the escape velocity, in the present work they are calculated like longitudinal light round-trip effects $\left(t(r)=t_{0}\left(1-(G M / r) / c^{2}\right)^{-1}\right)$, where $G M / r$ is the square of the implicit velocity in the earth's Keplerian velocity field. To first approximation, the effect, predicted by both approaches, is proportional to $(G M / r) / c^{2}$. On the earth-surface these effects are extremely small $10^{-10}$ and, excepting Mössbauer resonance scatterings, are very difficult to detect.

\subsection{The Excess Time Delay of Radar Signals in Round-Trips within the Solar System}

According to GR, massive astronomical bodies cause curvature of the spacetime geometry in their neighborhood as given by Equation (3), of the Introduction. This spacetime curvature symmetrically lengthens the go-return path of electromagnetic (EM) signals passing by these massive bodies and giving rise to an excess time delay. However, according to the present work the excess time delay is not caused by increase of the geometrical distance, however is due to the effective velocity of the signals in the Keplerian velocity field round the sun. In the case of round-trips between earth and Venus, the solar velocity field is favorable during the prograde travel, increasing the effective signal velocity and reducing the travel time. It however is unfavorable during the retrograde travels, decreasing the effective signal velocity and increasing the return travel time. The problem is totally analogous to that of light round-trips between two mirrors within a laboratory, moving with respect to the local HQS. Within the solar Keplerian velocity field, the calculation however is a little bit complicated, because the velocity of the signal depends on the position.

In the present work, the excess or deficit of time delay was numerically calculated along straight line paths, separately for travels from earth to Venus and back travel from Venus to earth in terms of the effective velocity $v_{\mathrm{eff}}=\boldsymbol{c}_{\mathrm{HQS}}+\boldsymbol{V}$, where $\boldsymbol{c}_{\mathrm{HQS}}$ is the fixed velocity of light with respect to the local HQS. This calculation was repeated for a series of paths, having minimum distances from the center of the sun $R=2,4,8,25,50$ and 100 million $\mathrm{km}$, before and after superior conjunction. The excess time delays were calculated dividing the straight line paths into about 420 segments, shorter segments near to the sun, separately for transverse and longitudinal components of the solar Keplerian velocity field along the signal paths and finally adding up the contributions along the full path. More than $99 \%$ of the excess time-loss in the retrograde journey or time-gain in the prograde journey comes from the longitudinal component of the solar velocity field along the path. The different Earth-Venus distances, due to the orbital 
positions as well as the slightly different signal path, due to the motion of earth during each signal round-trip, was taken into account. The obtained results are given in Table 1 and plotted in Figure 3.

The fact that the Keplerian velocity field of the HQS round the sun correctly produces the effective excess time delay (Shapiro effect) fully corroborates the present HQS-dynamics gravitational mechanism.

\subsection{The Gravitational Light Lensing Effect}

Consider two light beams, coming from two distant stars and propagating directly toward the sun as shown in Figure 4. First, in the region where the velocity of light $c$ has almost only radial components, the wave vectors of the two beams will be refracted according to Equation (8a) by a total angle $+\alpha$, thereby the light beams gaining a small velocity component $V(r)=-(G M / r)^{1 / 2}$ along $-\phi$, which compensates for the drag by the solar velocity field. This reduces the radial velocity component of light to $c^{\prime}=\left(c_{\mathrm{HQS}}^{2}-V^{2}\right)^{1 / 2}$. Near to the sun, where the solar velocity field achieves $436 \mathrm{~km} / \mathrm{sec}$ and is mostly parallel (left) and anti-parallel (right) to the beams, refraction by Equation (8b) is dominant and the wave vectors are refracted oppositely by a total angle $-2 \alpha$. Finally, after having passed by the sun and going away in the opposite side, the wave vectors again are refracted according to Equation (8a) by nearly an angle $+\alpha$. However, near to the sun, the solar velocity field is favorable to the prograde ray (left side of Figure 4) so that it spends less time near to the sun and hence is deflected by a smaller angle $-(2 \alpha-\delta)$. To the retrograde ray however (right) the solar velocity

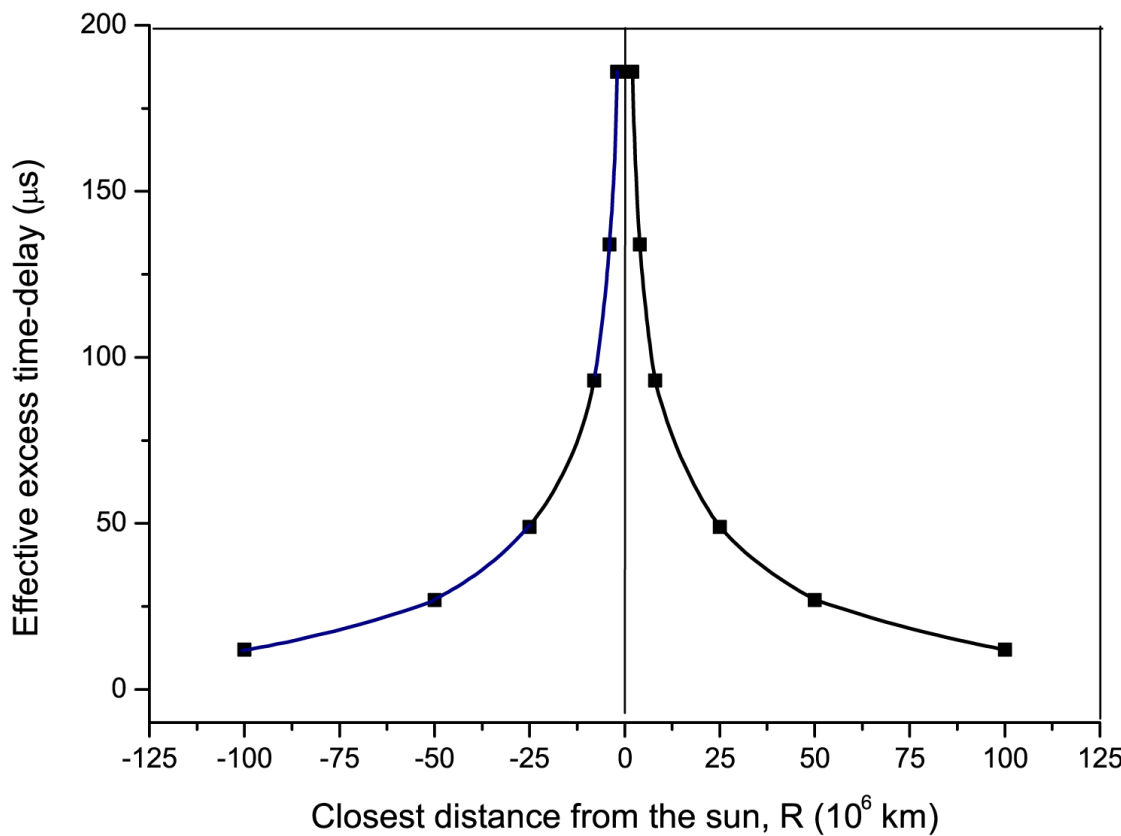

Figure 3. The calculated effective excess time delay, given in microseconds ( $\mu$ s), (fourth column in Table 1) for complete go-return round trips. The calculated data curves practically coincide with those, obtained by Shapiro [29]. 


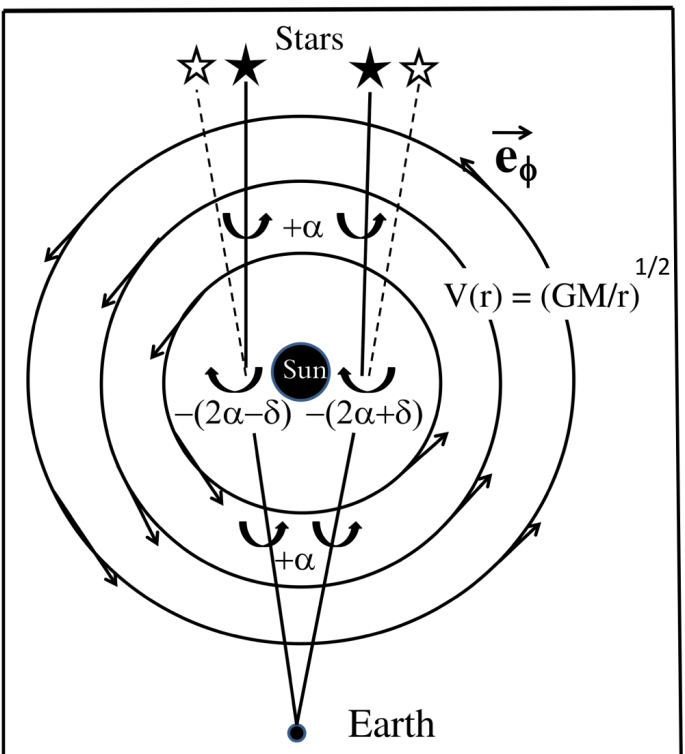

Figure 4. Gravitational light-lensing effect, by a heavy mass (Sun).

Table 1. The first column gives the values of the nominal impact parameters $R$, the second and third columns give respectively the gain of time (negative) and the loss of time (positive), due to the solar Keplerian velocity field of the HQS in milliseconds (ms) before and after superior conjunction. The forth column gives the effective excess time-delays for full go-return round-trips in microseconds $(\mu s)$.

\begin{tabular}{cccc}
\hline$R\left(10^{6} \mathrm{~km}\right.$ units $)$ & go: $\Delta t(\mathrm{~ms})$ & return: $\Delta t(\mathrm{~ms})$ & $\Delta t_{\text {eff }}(\mu \mathrm{s})$ \\
\hline 100 & -51.987 & 51.999 & 12 \\
50 & -76.697 & 76.725 & 28 \\
25 & -70.007 & 70.056 & 49 \\
8 & -48.585 & 48.679 & 94 \\
4 & -36.699 & 36.833 & 134 \\
2 & -27.165 & 27.351 & 186 \\
-2 & 27.351 & -27.165 & 186 \\
-4 & 36.833 & -36.699 & 134 \\
-8 & 48.679 & -48.585 & 94 \\
-25 & 70.056 & -70.007 & 49 \\
-50 & 76.725 & -76.697 & 28 \\
-100 & 51.999 & -51.987 & 12 \\
\hline
\end{tabular}

field is unfavorable and hence it spends a longer time near to the sun and refracts by a larger angle $-(2 \alpha+\delta)$. This differentiated refraction by $\delta$, due to Equation (8b) causes a (symmetric) convergence of the rays that is responsible for the gravitational light lensing effect.

The value of $\delta$ can be calculated simply by multiplying the refraction rate Equation (8b) times the excess (or shortage) of time delays, listed in Table 1. On 
the solar surface $R=6.9565 \times 10^{8} \mathrm{~m}$ and the refraction rate, given by Equation (8b), is $W_{\phi} \sim 0.0359 \mathrm{deg} / \mathrm{sec}$. Interpolating the value of $\Delta t$ in Table 1 for light passing by the surface of the sun, we find the excess time delay at the retrograde side or the shortage of time at the prograde side of closely $\Delta t=+13.5 \mathrm{~ms}$. The product $W_{\phi} \times \Delta t$ gives $\delta=1.745 \mathrm{arcsec}$, which is very closely the observed deflection angle, causing the convergence of light passing close by the solar surface.

\subsection{The Non-Synchronous Arrival of the Pulsar Signals to Equidistant Earth-Based Antennas along the Earth's Orbital Motion}

Astronomical observations with the help of interferometric methods, superposing coherently the signals of the digital images with the help of computers, can improve the images by orders of magnitude. To this end, it is necessary that the signals from the different receivers be synchronous, which is achieved by time keeping the signals with clocks closely synchronized with the help of the GPS clocks. However, on testing the so synchronized clocks by the arrival of the millisecond pulsar signals, a surprise. While the arrival of the pulsar signals to the antenna arrays along directions transverse to the earth's orbital motion was synchronous, along the orbital motion of earth the arrival was out of synchrony, up to $4.2 \mu \mathrm{sec}$ [19] [20].

The aberration of star light is an old and well known feature in astronomical observations from earth. Usually the aberration angle is explained in terms of the orbital velocity of earth $\sin \beta=V / c$. However, according to the present work, this aberration contains an additional feature that arises from the refraction rate Equation (8a) of light propagating from distant objects toward the sun. This refraction rate creates a velocity component of the light signal toward $-\phi$ and thereby reduces the radial velocity component to $c^{\prime}=\left(c_{\mathrm{HQS}}^{2}-V^{2}\right)^{1 / 2}$. Correspondingly it slants the wave fronts by and angle $\beta$, so that the wave fronts arrive earth inclined, reaching first the leading part of earth and only $4.2 \mu \mathrm{sec}$ later the rear part. Note that GR too predicts reduction of the velocity of light, propagating toward the sun. This reduction however is imputed to stretching of the radial coordinate and not to effective velocity.

\subsection{The Perihelion Precession}

An analogous differentiated refraction rate of the propagation velocity $\boldsymbol{v}$ of an orbiting body as in the preceding Section 5.6 must be responsible for the perihelion precession of elliptical orbits. At the aphelion the direction of the effective velocity $\boldsymbol{v}_{\text {eff }}$, with respect to the HQS, is opposite (retrograde) to the velocity field of the HQS as well as to the orbital motion. This increases the time of permanence in this region of the orbit. As the refraction rate is a characteristic of the position, the effective velocity vector of the particle $v_{\text {eff }}$ refracts during a longer time. However, at the perihelion $v_{\text {eff }}$ is parallel to the velocity field (prograde) and to the orbital velocity, which displaces it more 
rapidly (than the HQS), so that it has not time enough to recover the tangential direction. It recovers it only somewhat beyond the ideal perihelion point. In this way the perihelion advances a little bit in the prograde sense in each orbital round-trip.

\subsection{Absence of Effects of the Solar Gravitational Field on the GPS Clocks}

In the view of the present HQS-dynamics gravitational mechanism, the slowing of clocks is caused by velocity with respect to the local HQS (and with respect to the local LFs) and not by relative velocity. Hence, clocks, stationary with respect to the local moving HQS, do not run slow. They show proper time. The effective velocity of the GPS clocks with respect to the local moving HQS, commoving with earth round the sun, is zero, given by:

$$
\boldsymbol{v}_{\text {eff }}=\boldsymbol{V}(r)-\boldsymbol{v}_{\text {orb }} \cong(G M / r)^{1 / 2} \boldsymbol{e}_{\phi}-(G M / r)^{1 / 2} \boldsymbol{e}_{\phi} \approx 0
$$

All clocks orbiting in circular equatorial orbits round an astronomical body, which normally is moving itself in a regular circular equatorial orbit round a larger body (star or galaxy), are stationary with respect to the local HQS. Such clocks all are naturally synchronous with respect to each other and all show closely the same proper time throughout the universe.

\subsection{Effects of the Velocity Field of the HQS Equation (6) on Clocks Moving in Non-Equatorial Circular Orbits}

AS discussed in Section 2, the GPS satellites move at $2.02 \times 10^{4} \mathrm{~km}$ of altitude, along circular orbits inclined 55 degrees with respect to the earth's equator and hence have a considerable velocity with respect to the local HQS in the Keplerian velocity field, creating the earth's gravitational field. The GPS clocks have velocity components, given by $v_{0}(1-\cos \alpha)$ along $-\phi$ and $v_{0} \sin \alpha$ along $\pm \theta$, where $v_{0}=3.87 \mathrm{~km} / \mathrm{sec}$ and $\alpha$ is the angle of the orbital velocity $v_{0}$ with respect to the equator or parallels. The effective velocity at the equator is $v_{0}[2(1-\cos \alpha)]^{1 / 2}=3.574 \mathrm{~km} / \mathrm{sec}$ and the estimated average velocity of the GPS satellites with respect to the local HQS over the entire orbit is

$\sim 0.8 \times 3.574=2.86 \mathrm{~km} / \mathrm{sec}$. Analogously the Westward velocity of the earth-based station at Colorado highs with respect to the local HQS is about $7.4 \mathrm{~km} / \mathrm{sec}$. In addition, the velocity of the Cs atoms of $0.255 \mathrm{~km} / \mathrm{sec}$ within the atomic clocks as well as a small transverse Doppler shift, due to the implicit velocity of the earth-based stations with respect to the local HQS must be considered. Altogether these effects achieve closely the observed $4.5 \times 10^{-10}$ $\sec / \sec [3]$.

\subsection{The Astronomical Motions Closely Track the Motion of the HQS throughout the Universe}

The null results of the light anisotropy experiments, due to the orbital and cos- 
mic motion of earth and the absence of the gravitational time dilation on the GPS clocks, due to the solar gravitational field, proves that the planet earth is stationary with respect to the local HQS that rules the propagation of light. From the HQS-dynamics point of view, the planetary orbits lie all closely within the equatorial plane of the solar Keplerian velocity field of the HQS and the orbit of the solar system lies closely within the galactic disk, which is the equatorial plane of the galactic velocity field of the HQS. The reason is that this configuration of the orbital motions minimizes the velocity of these astronomical bodies with respect to the local HQS, which is the result of the wavelength stretching of particles and light. Wavelength stretching reduces the kinetic energy of particles with respect to the local HQS, and reduces the energy of radiation, as is well known from the cosmic microwave background radiation. However the averaging down of velocity, during gravitational agglomeration into large matter bodies too has very significantly contributed. Observations indicate that not only the planets of the solar system, not only the solar system in the Milky-Way galaxy however astronomical bodies in general throughout the universe are very closely stationary with respect to the local HQS in the respective gravitational fields, which predicts the universality of the laws of physics.

\subsection{Black-Hole Singularities}

From the present HQS-dynamics point of view, black holes are Keplerian velocity fields of the HQS in which the velocity of the HQS along the $+\phi$ spherical coordinate, achieves velocities equal to the velocity of light $V(r)=c$. In this velocity field, the radial component of the velocity of a light signal, propagating directly toward the black-hole, falls gradually and effectively becomes zero at the event horizon. The reason for this reduction is the fact that, due to the refraction rate Equation (8a), light develops an orthogonal implicit velocity component along $-\phi$, that achieves itself the velocity of light. Also in the view of an external observer, a clock, stationary at the event horizon $r_{g}$, will seem to be stopped, because the time standard, by which it counts time, will take an infinite time to complete one period of oscillation. In GR the velocity of light propagating directly toward the black-hole too falls gradually to zero. However the reason is completely different. It is the increasing length of the geometrical distances along the radial coordinate that tend to infinity. GR, instead of slowing the velocity component, stretches the street.

From the present HQS dynamics viewpoint, the event horizon of black-holes is anisotropic. For light propagating along $-\phi$ the escape velocity is given by $c-V=\sqrt{2} V$, which means that the event horizon lies at a radial distance where the velocity of the local HQS is $V=0.4142 c$ and that the event horizon is a spherical surface, located at $r=2.9144 r_{g}$, where $r_{g}$ is the gravitational radius of the black-hole, from the viewpoint of GR. However, a light signal, propagating along $+\phi$, has an ordinary (orbital) velocity $c+V$ that achieves $2 c$ at the conventional event horizon and thus can escape along an outward hyperbolic 
path. For such a light pulse the event horizon lies lower than $r_{g}$, defined by $c+V=\sqrt{2} V$, where $V=2.4142 c$ and the event horizon is a spherical surface located at $r=0.08578 r_{g}$. Above this event horizon, the direct orbit of light becomes outward elliptic and eventually hyperbolic. From the present view, a black-hole is black at the retrograde side and is bright at the prograde side.

\section{Final Comments and Conclusions}

This work has discussed the problems with Einstein's assumptions in the special and general theories of relativity and showed their correct solution within the Higgs quantum space (HQS) dynamics. The Higgs theory introduces profound changes in Einstein's view about the empty space (vacuum) and the meaning of motions. The HQS constitutes not only a local ultimate (locally absolute) reference for rest and for motions of matter-energy; however literally governs these motions. It materializes the local Lorentz frames (LFs) turning them into local proper LFs, intrinsically stationary with respect to the local HQS and thus moving with it in its motions. Motions with respect to the local HQS (LFs) and not relative motions are responsible for all effects of motion. However, Lorentz frames moving with respect to the local HQS (LFs) are not proper LFs.

In the scenario of the Higgs theory, the local HQS (local proper LFs) can itself move in the ordinary three-dimensional space, thereby giving rise to implicit motions for matter bodies, stationary in the ordinary space. Therefore, the present work associates together the central idea of the Higgs theory, according to which the HQS governs the inertial motion of matter-energy and the central idea of GR, according to which the gravitational dynamics is an inertial dynamics and replaces Einstein's static curved spacetime by a Keplerian velocity field $\boldsymbol{V}(r)=(G M / r)^{1 / 2} \boldsymbol{e}_{\phi}$ of the HQS. This Keplerian velocity field of the HQS is the quintessence of the gravitational fields. It has the crucial property of giving rise to the outside inside centrifuge phenomenon, observed within gravitational fields, in which the gravitational pull is identically a centrifugal pull. It causes hyperbolic rotation of the implicit $r$ and $\phi$ velocity components (with respect to the local HQS); that is shown to appropriately create the observed gravitational dynamics and all the observed effects of the gravitational fields on light and on clocks.

Viewing that the gravitational dynamics of the astronomical systems throughout the universe (planetary satellite systems, solar system, galaxies etc.) all are governed by velocity field of the HQS, moving concomitantly with the astronomical bodies, these bodies all are very nearly stationary with respect to the local HQS, which directly leads to the universality of the laws of physics.

\section{References}

[1] Von Laue, M. (1955) Annalen der Physik, 38.

[2] Lorentz, H.A., Einstein, A., Minkowski, H. and Weyl, H. (1923) The Principle of 
Relativity. Dover Publications, New York.

[3] Ashby, N. (1996) Mercury, 23-27.

[4] Bailey, H., Borer, K., Combley, F., Drumm, H. and Krienen, F. (1977) Nature, 268, 301-305. https://doi.org/10.1038/268301a0

[5] Rubin, V. and Ford Jr., W.K. (1970) The Astrophysical Journal, 159, 379. https://doi.org/10.1086/150317

[6] Rubin, V., Thonnard, N. and Ford Jr., W.K. (1980) The Astrophysical Journal, 238, 471-487. https://doi.org/10.1086/158003

[7] Riess, A., et al. (1998) The Astrophysical Journal, 116, 1009.

[8] Perlmutter, S., et al. (1999) The Astrophysical Journal, 517, 565. https://doi.org/10.1086/307221

[9] Schaf, J. (2017) The True Origin of the Gravitational Dynamics. Scientific Research Publishing, Inc., USA.

[10] Schaf, J. (2017) World Journal of Research and Review, 4, 68.

[11] Schaf, J. (2017) International Journal of Advanced Research in Physical Science, 4, 1.

[12] Anderson, P.W. (1963) Physical Review, 130, 439. https://doi.org/10.1103/PhysRev.130.439

[13] Ginzburg, V.L. and Landau, L.D. (1950) Journal of Experimental and Theoretical Physics (JETP), 20, 1064.

[14] Meissner, W. and Ochsenfeld, R. (1933) Naturwissenschaften, 21, 787-788. https://doi.org/10.1007/BF01504252

[15] Higgs, P.W. (1964) Physical Review Letters, 13, 508. https://doi.org/10.1103/PhysRevLett.13.508

[16] Englert, F. and Brout, R. (1964) Physical Review Letters, 13, 321. https://doi.org/10.1103/PhysRevLett.13.321

[17] Carrol, S.M. (2001) Living Reviews in Relativity, 4, 1. https://doi.org/10.12942/lrr-2001-1

[18] Sola, J. (2013) Journal of Physics: Conference Series, 453, Article ID: 012015. https://doi.org/10.1088/1742-6596/453/1/012015

[19] Hatch, R.R. (2004) GPS Solutions, 8, 67-73. https://doi.org/10.1007/s10291-004-0092-8

[20] Hatch, R.R. (2004) Foundations of Physics, 34, 1725-1739. https://doi.org/10.1007/s10701-004-1313-2

[21] Hatch, R.R. (2007) Physics Essays, 20, 83. https://doi.org/10.4006/1.3073811

[22] Schaf, J. (2018) Journal of Modern Physics, 9, 395-418. https://doi.org/10.4236/jmp.2018.93028

[23] Schaf, J. (2015) Universal Journal of Physics and Applications, 9, 141.

[24] Schaf, J. (2014) Recent Progress in Space Technology, 4, 44.

[25] Schaf, J. (2014) Journal of Modern Physics, 5, 407-448. https://doi.org/10.4236/jmp.2014.56053

[26] Miller, D.C. (1933) Review of Modern Physics, 5, 203. https://doi.org/10.1103/RevModPhys.5.203

[27] Pound, R.V. and Snider, J.L. (1965) Physical Review B, 140, B788. 
https://doi.org/10.1103/PhysRev.140.B788

[28] Brault, J.W. (1963) Bulletin of the American Physical Society, 8, 28.

[29] Shapiro, I.I., et al. (1971) Physical Review Letters, 26, 1132.

https://doi.org/10.1103/PhysRevLett.26.1132 